\title{
A Delay-Dependent Approach to Stability of Uncertain Discrete-Time State-Delayed Systems with Generalized Overflow Nonlinearities
}

\author{
V. Krishna Rao Kandanvli and Haranath Kar \\ Department of Electronics and Communication Engineering, Motilal Nehru National Institute of Technology, Allahabad 211004, India \\ Correspondence should be addressed to V. Krishna Rao Kandanvli, krishnaraonit@yahoo.co.in
}

Received 26 May 2012; Accepted 10 October 2012

Academic Editors: G. Bella, A. Dujella, M.-B. Hu, and R. López-Ruiz

Copyright ( 2012 V. K. R. Kandanvli and H. Kar. This is an open access article distributed under the Creative Commons Attribution License, which permits unrestricted use, distribution, and reproduction in any medium, provided the original work is properly cited.

This paper addresses the problem of global asymptotic stability of a class of uncertain discrete-time state-delayed systems employing generalized overflow nonlinearities. The systems under investigation involve parameter uncertainties that are assumed to be deterministic and norm bounded. A new computationally tractable delay-dependent criterion for global asymptotic stability of such systems is presented. A numerical example is given to illustrate the effectiveness of the proposed method.

\section{Introduction}

In the implementation of linear discrete systems, signals are usually represented and processed in a finite wordlength format which frequently generates several kinds of nonlinearities, such as overflow and quantization. Such nonlinearities may lead to instability in the designed system. Therefore, the study of stability problem for discrete-time systems with finite wordlength nonlinearities is important not only for its theoretical interest but also for application to practical system design. Many publications [1-22] relating to the issue of the global asymptotic stability of discrete-time systems with overflow nonlinearities have appeared.

Parameter uncertainties are often introduced in many physical systems as a consequence of variations in system parameters, modeling errors or some ignored factors. Such uncertainties may result in the deterioration of system performance and instability of the system.

Time delay is another source of instability for discretetime systems. They are frequently introduced in many physical, industrial, and engineering systems due to finite capabilities of information processing and signal transmission among various parts of the system. During the past few decades, there has emerged a considerable interest on the stability analysis problems for delayed systems [17-21, 2331]. According to the dependence of delay, the available stability criteria for delayed systems can be broadly classified into two types: delay independent and delay dependent. Increasing attention is being paid to delay-dependent stability criteria for delayed systems since they can often provide less conservative results than delay-independent criteria [17, $25,27]$.

The problem of establishing delay-dependent criteria for the global asymptotic stability of discrete-time uncertain state-delayed systems with overflow nonlinearities is an important and challenging task. So far, very little attention has been paid for the investigation of this problem $[17,21]$.

In this paper, we consider the problem of global asymptotic stability of a class of discrete-time uncertain statedelayed systems employing generalized overflow nonlinearities. The system under investigation involves parameter uncertainties that are assumed to be norm-bounded. The paper is organized as follows. Section 2 presents a description of the system under consideration. New computationally tractable delay-dependent criteria for global asymptotic stability of uncertain discrete-time state-delayed systems employing generalized overflow nonlinearities are proposed in Section 3. In Section 4, a comparison of the proposed 
method with [18] is made. It is shown that the result presented in [18] is recovered from the presented approach as a special case. A numerical example illustrating the applicability of the proposed criterion is given in Section 5 .

\section{System Description}

The class of nonlinear discrete-time uncertain state-delayed systems under consideration is given by

$$
\begin{aligned}
& \mathbf{x}(k+1)=\mathbf{f}(\mathbf{y}(k)) \\
& =\left[\begin{array}{llll}
f_{1}\left(y_{1}(k)\right) & f_{2}\left(y_{2}(k)\right) & \cdots & f_{n}\left(y_{n}(k)\right)
\end{array}\right]^{T}, \\
& \mathbf{y}(k)=(\mathbf{A}+\Delta \mathbf{A}) \mathbf{x}(k)+\left(\mathbf{A}_{\mathbf{d}}+\Delta \mathbf{A}_{\mathbf{d}}\right) \mathbf{x}(k-d(k)) \\
& =\left[\begin{array}{llll}
y_{1}(k) & y_{2}(k) & \cdots & y_{n}(k)
\end{array}\right]^{T},
\end{aligned}
$$

where $\mathbf{x}(k) \in \mathbf{R}^{n}$ is the state vector, $\mathbf{A}, \mathbf{A}_{\mathbf{d}} \in \mathbf{R}^{n \times n}$ are the known constant matrices and $\Delta \mathbf{A}, \Delta \mathbf{A}_{\mathbf{d}} \in \mathbf{R}^{n \times n}$ are assumed to be of the usual norm-bounded type as follows:

$$
\left[\begin{array}{ll}
\Delta \mathrm{A} & \Delta \mathrm{A}_{\mathrm{d}}
\end{array}\right]=\mathrm{BF}\left[\begin{array}{ll}
\mathrm{C}_{0} & \mathrm{C}_{1}
\end{array}\right]
$$

where $\mathbf{B} \in \mathbf{R}^{n \times l}, \mathbf{C}_{\mathbf{0}}, \mathbf{C}_{\mathbf{1}} \in \mathbf{R}^{m \times n}$ are known matrices representing the structure of uncertainty and $\mathbf{F} \in \mathbf{R}^{l \times m}$ is an unknown matrix which satisfies

$$
\mathbf{F}^{T} \mathbf{F} \leq \mathbf{I}
$$

It may be mentioned that the uncertainty structure of (2a) and (2b) has been widely adopted in robust control and filtering for uncertain systems [32-34]. $\{\varphi(k), k=$ $\left.-d_{2},-d_{2}+1, \ldots, 0\right\} \in \mathbf{R}^{n}$ is the initial state value at time $k$ and $\mathbf{f}(\cdot)$ represents the generalized overflow nonlinearities.

The generalized overflow characteristic is given by (see Figure 1):

$$
\left.\begin{array}{cc}
L \leq f_{i}\left(y_{i}(k)\right) \leq 1, & y_{i}(k)>1, \\
f_{i}\left(y_{i}(k)\right)=y_{\mathrm{i}}(k), & -1 \leq y_{i}(k) \leq 1, \\
-1 \leq f_{i}\left(y_{i}(k)\right) \leq-\mathrm{L}, \quad y_{i}(k)<-1,
\end{array}\right\} \quad i=1,2, \ldots, n,
$$

where

$$
-1 \leq L \leq 1
$$

With appropriate choice of $L,(3 a)$ and (3b) represent the usual types of overflow arithmetics employed in practice, such as saturation $(L=1)$, zeroing $(L=0)$, two's complement $(L=-1)$, and triangular $(L=-1)$. The timevarying delay $d(k)$, known as range-like or interval-like timevarying delay $[28,30,31]$, satisfying

$$
d_{1} \leq d(k) \leq d_{2}
$$

where $d_{1}$ and $d_{2}$ are known positive integers representing the lower and upper delay bounds, respectively. Such delays

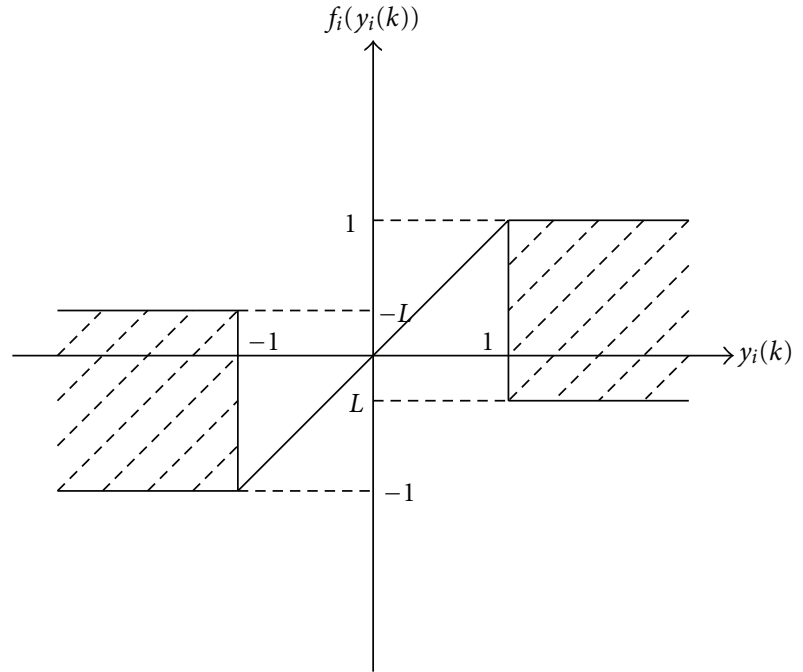

FIgURE 1: The generalized overflow nonlinearity described by (3a) and (3b).

may be used to characterize the realistic situation in many practical applications $[28,31,35,36]$.

The purpose of this paper is to develop delay-dependent robust stability criteria for the system (1a) and (1b)-(3a) and (3b) for any interval like time-varying delay $d(k)$ satisfying (4).

\section{Main Results}

In this section, delay-dependent criteria for the global asymptotic stability of system (1a) and (1b)-(4) are established.

The following lemmas are needed in the proof of our main results.

Lemma 1 (see [8]). An $n \times n$ positive definite symmetric matrix $\mathbf{H}=\left[h_{i j}\right]$ satisfies

$$
\mathbf{y}^{T}(k) \mathbf{H y}(k)-\mathbf{f}^{T}(\mathbf{y}(k)) \mathbf{H f}(\mathbf{y}(k)) \geq 0,
$$

if and only if

$$
(1+L) h_{i i} \geq 2 \sum_{j=1, j \neq i}^{n}\left|h_{i j}\right|, \quad i=1,2, \ldots, n .
$$

Lemma 2 (see [32-34, 37]). Let $\mathbf{\Sigma}, \boldsymbol{\Gamma}, \mathbf{F}$ and $\mathbf{M}$ be real matrices of appropriate dimensions with $\mathbf{M}$ satisfying $\mathbf{M}=\mathbf{M}^{T}$, then

$$
\mathbf{M}+\boldsymbol{\Sigma} \mathbf{F} \boldsymbol{\Gamma}+\boldsymbol{\Gamma}^{T} \mathbf{F}^{T} \boldsymbol{\Sigma}^{T}<\mathbf{0},
$$

for all $\mathbf{F}^{T} \mathbf{F} \leq \mathbf{I}$, if and only if there exists a scalar $\varepsilon>0$ such that

$$
\mathbf{M}+\varepsilon^{-1} \boldsymbol{\Sigma} \boldsymbol{\Sigma}^{T}+\varepsilon \boldsymbol{\Gamma}^{T} \boldsymbol{\Gamma}<\mathbf{0} .
$$

Next, we present the main results of the paper. 
Theorem 3. For given positive integers $d_{1}$ and $d_{2}$ with $d_{2} \geq$ $d_{1}$, the system described by (1a) and (1b)-(4) is globally asymptotically stable if there exists appropriately dimensioned matrices $\mathbf{P}=\mathbf{P}^{T}>\mathbf{0}, \mathbf{Q}_{\mathbf{i}}=\mathbf{Q}_{\mathbf{i}}^{T}>\mathbf{0}(i=1,2,3), \mathbf{Z}_{\mathbf{i}}=\mathbf{Z}_{\mathbf{i}}^{T}>$ $\mathbf{0}(i=1,2), \mathbf{H}=\mathbf{H}^{T}=\left[h_{i j}\right]>\mathbf{0}, \mathbf{X}=\left[\begin{array}{ll}\mathbf{X}_{11} & \mathbf{X}_{12} \\ \mathbf{X}_{12}^{T} & \mathbf{X}_{22}\end{array}\right] \geq \mathbf{0}$, $\mathbf{Y}=\left[\begin{array}{ll}\mathbf{Y}_{11} & \mathbf{Y}_{12} \\ \mathbf{Y}_{12}^{T} & \mathbf{Y}_{22}\end{array}\right] \geq \mathbf{0}, \mathbf{N}=\left[\begin{array}{l}\mathbf{N}_{1} \\ \mathbf{N}_{2}\end{array}\right], \mathbf{M}=\left[\begin{array}{l}\mathbf{M}_{1} \\ \mathbf{M}_{2}\end{array}\right], \mathbf{S}=\left[\begin{array}{l}\mathbf{S}_{1} \\ \mathbf{S}_{2}\end{array}\right]$ and a positive scalar $\varepsilon$ such that

$$
(1+L) h_{i i} \geq 2 \sum_{j=1, j \neq i}^{n}\left|h_{i j}\right|, \quad i=1,2, \ldots, n,
$$

and the following (10)-(13) hold,

$$
\begin{gathered}
{\left[\begin{array}{ccccccc}
\xi_{11} & \xi_{12} & \mathbf{S}_{1} & -\mathbf{M}_{1} & -\bar{Z} & \mathbf{A}^{T} \mathbf{H} & \mathbf{0} \\
\xi_{12}^{T} & \xi_{22} & \mathbf{S}_{2} & -\mathbf{M}_{2} & \mathbf{0} & \mathbf{A}_{\mathbf{d}}^{T} \mathbf{H} & \mathbf{0} \\
\mathbf{S}_{1}^{T} & \mathbf{S}_{2}^{T} & -\mathbf{Q}_{1} & \mathbf{0} & \mathbf{0} & \mathbf{0} & \mathbf{0} \\
-\mathbf{M}_{1}^{T} & -\mathbf{M}_{2}^{T} & \mathbf{0} & -\mathbf{Q}_{2} & \mathbf{0} & \mathbf{0} & \mathbf{0} \\
-\overline{\mathbf{Z}} & \mathbf{0} & \mathbf{0} & \mathbf{0} & \mathbf{P}-\mathbf{H}+\overline{\mathbf{Z}} & \mathbf{0} & \mathbf{0} \\
\mathbf{H A} & \mathbf{H A}_{\mathbf{d}} & \mathbf{0} & \mathbf{0} & \mathbf{0} & -\mathbf{H} & \mathbf{H B} \\
\mathbf{0} & \mathbf{0} & \mathbf{0} & \mathbf{0} & \mathbf{0} & \mathbf{B}^{T} \mathbf{H} & -\varepsilon \mathbf{I}
\end{array}\right]<\mathbf{0}} \\
\psi_{\mathbf{1}}=\left[\begin{array}{cc}
\mathbf{X} & \mathbf{N} \\
\mathbf{N}^{T} & \mathbf{Z}_{1}
\end{array}\right] \geq \mathbf{0} \\
\psi_{\mathbf{2}}=\left[\begin{array}{cc}
\mathbf{Y} & \mathbf{S} \\
\mathbf{S}^{T} & \mathbf{Z}_{2}
\end{array}\right] \geq \mathbf{0} \\
\psi_{3}
\end{gathered}
$$

where

$$
\begin{gathered}
\overline{\mathbf{Z}}=d_{2} \mathbf{Z}_{1}+\left(d_{2}-d_{1}\right) \mathbf{Z}_{2} \\
\xi_{11}=-\mathbf{P}+\mathbf{Q}_{1}+\mathbf{Q}_{2}+\left(d_{2}-d_{1}+1\right) \mathbf{Q}_{3}+\overline{\mathbf{Z}}+\mathbf{N}_{\mathbf{1}}+\mathbf{N}_{1}^{T} \\
+d_{2} \mathbf{X}_{11}+\left(d_{2}-d_{1}\right) \mathbf{Y}_{11}+\varepsilon \mathbf{C}_{0}^{T} \mathbf{C}_{0} \\
\boldsymbol{\xi}_{12}=-\mathbf{N}_{\mathbf{1}}+\mathbf{N}_{2}^{T}+\mathbf{M}_{\mathbf{1}}-\mathbf{S}_{\mathbf{1}}+d_{2} \mathbf{X}_{12}+\left(d_{2}-d_{1}\right) \mathbf{Y}_{12} \\
+\varepsilon \mathbf{C}_{0}^{T} \mathbf{C}_{1}, \\
\xi_{22}=-\mathbf{N}_{2}-\mathbf{N}_{2}^{T}+\mathbf{M}_{2}+\mathbf{M}_{2}^{T}-\mathbf{S}_{2}-\mathbf{S}_{2}^{T}-\mathbf{Q}_{3} \\
+d_{2} \mathbf{X}_{22}+\left(d_{2}-d_{1}\right) \mathbf{Y}_{22}+\varepsilon \mathbf{C}_{1}^{T} \mathbf{C}_{\mathbf{1}}
\end{gathered}
$$

Proof. Let

$$
\begin{gathered}
\boldsymbol{\eta}(k)=\mathbf{x}(k+1)-\mathbf{x}(k), \\
=\mathbf{f}(\mathbf{y}(k))-\mathbf{x}(k) .
\end{gathered}
$$

Consider a quadratic Lyapunov function [23]

$$
\begin{aligned}
& v(\mathbf{x}(k))= v_{1}(\mathbf{x}(k))+v_{2}(\mathbf{x}(k))+v_{3}(\mathbf{x}(k)) \\
&+v_{4}(\mathbf{x}(k)), \\
& v_{1}(\mathbf{x}(k))=\mathbf{x}^{T}(k) \mathbf{P} \mathbf{x}(k), \\
& v_{2}(\mathbf{x}(k))=\sum_{\theta=-d_{2}+1 l=\theta-1}^{0} \sum^{-1} \boldsymbol{\eta}^{T}(k+l) \mathbf{Z}_{1} \boldsymbol{\eta}(k+l) \\
&+\sum_{\theta=-d_{2}+1 l=\theta-1}^{-d_{1}} \sum^{T}(k+l) \mathbf{Z}_{2} \boldsymbol{\eta}(k+l), \\
& v_{3}(\mathbf{x}(k))=\sum_{l=-d_{1}}^{-1} \mathbf{x}^{T}(k+l) \mathbf{Q}_{1} \mathbf{x}(k+l) \\
&+\sum_{l=-d_{2}}^{-1} \mathbf{x}^{T}(k+l) \mathbf{Q}_{2} \mathbf{x}(k+l) \\
& \sum_{\theta=-d_{1}+1}^{-1} \sum_{d_{2}+1 l=\theta-1}^{-1} \mathbf{x}^{T}(k+l) \mathbf{Q}_{3} \mathbf{x}(k+l)
\end{aligned}
$$

Defining

$$
\Delta v(\mathbf{x}(k))=v(\mathbf{x}(k+1))-v(\mathbf{x}(k)),
$$

yields

$$
\begin{aligned}
& \Delta v_{1}(\mathbf{x}(k))=\mathbf{x}^{T}(k+1) \mathbf{P} \mathbf{x}(k+1)-\mathbf{x}^{T}(k) \mathbf{P} \mathbf{x}(k) \\
& =\mathbf{f}^{T}(\mathbf{y}(k)) \mathbf{P f}(\mathbf{y}(k))-\mathbf{x}^{T}(k) \mathbf{P x}(k), \\
& \Delta v_{2}(\mathbf{x}(k))=d_{2} \boldsymbol{\eta}^{T}(k) \mathbf{Z}_{\mathbf{1}} \boldsymbol{\eta}(k)-\sum_{\theta=-d_{2}}^{-1} \boldsymbol{\eta}^{T}(k+\theta) \mathbf{Z}_{\mathbf{1}} \boldsymbol{\eta}(k+\theta) \\
& +\left(d_{2}-d_{1}\right) \boldsymbol{\eta}^{T}(k) \mathbf{Z}_{2} \boldsymbol{\eta}(k) \\
& -\sum_{\theta=-d_{2}}^{-d_{1}-1} \boldsymbol{\eta}^{T}(k+\theta) \mathbf{Z}_{2} \boldsymbol{\eta}(k+\theta) \\
& =\boldsymbol{\eta}^{T}(k) \overline{\mathbf{Z}} \boldsymbol{\eta}(k)-\sum_{\theta=-d(k)}^{-1} \boldsymbol{\eta}^{T}(k+\theta) \mathbf{Z}_{\mathbf{1}} \boldsymbol{\eta}(k+\theta) \\
& -\sum_{\theta=-d(k)}^{-d_{1}-1} \boldsymbol{\eta}^{T}(k+\theta) \mathbf{Z}_{2} \boldsymbol{\eta}(k+\theta) \\
& -\sum_{\theta=-d_{2}}^{-d(k)-1} \boldsymbol{\eta}^{T}(k+\theta)\left(\mathbf{Z}_{\mathbf{1}}+\mathbf{Z}_{\mathbf{2}}\right) \boldsymbol{\eta}(k+\theta), \\
& \Delta v_{3}(\mathbf{x}(k))=\mathbf{x}^{T}(k)\left(\mathbf{Q}_{1}+\mathbf{Q}_{2}\right) \mathbf{x}(k)-\mathbf{x}^{T}\left(k-d_{1}\right) \mathbf{Q}_{1} \mathbf{x}\left(k-d_{1}\right) \\
& -\mathbf{x}^{T}\left(k-d_{2}\right) \mathbf{Q}_{2} \mathbf{x}\left(k-d_{2}\right),
\end{aligned}
$$




$$
\Delta v_{4}(\mathbf{x}(k))=\left(d_{2}-d_{1}+1\right) \mathbf{x}^{T}(k) \mathbf{Q}_{3} \mathbf{x}(k)
$$$$
-\sum_{\theta=-d_{2}}^{-d_{1}} \mathbf{x}^{T}(k+\theta) \mathbf{Q}_{3} \mathbf{x}(k+\theta)
$$$$
\leq\left(d_{2}-d_{1}+1\right) \mathbf{x}^{T}(k) \mathbf{Q}_{3} \mathbf{x}(k)
$$$$
-\mathbf{x}^{T}(k-d(k)) \mathbf{Q}_{3} \mathbf{x}(k-d(k)) .
$$

Using (15), we obtain the following null products [23]

$$
\begin{aligned}
& 0=2 \zeta_{\mathbf{1}}^{T}(k)\left[\begin{array}{ll}
\mathbf{N}_{\mathbf{1}}^{T} & \mathbf{N}_{2}^{T}
\end{array}\right]^{T} \\
& \times\left[\mathbf{x}(k)-\mathbf{x}(k-d(k))-\sum_{l=-d(k)}^{-1} \boldsymbol{\eta}(k+l)\right], \\
& 0=2 \zeta_{1}^{T}(k)\left[\begin{array}{ll}
\mathbf{M}_{1}^{T} & \mathbf{M}_{2}^{T}
\end{array}\right]^{T} \\
& \times\left[\mathbf{x}(k-d(k))-\mathbf{x}\left(k-d_{2}\right)-\sum_{l=-d_{2}}^{-d(k)-1} \boldsymbol{\eta}(k+l)\right], \\
& 0=2 \zeta_{1}^{T}(k)\left[\begin{array}{ll}
\mathbf{S}_{1}^{T} & \mathbf{S}_{2}^{T}
\end{array}\right]^{T} \\
& \times\left[\mathbf{x}\left(k-d_{1}\right)-\mathbf{x}(k-d(k))-\sum_{l=-d(k)}^{-d_{1}-1} \boldsymbol{\eta}(k+l)\right],
\end{aligned}
$$

where

$$
\zeta_{1}(k)=\left[\begin{array}{ll}
\mathbf{x}^{T}(k) & \mathbf{x}^{T}(k-d(k))
\end{array}\right]^{T} .
$$

For any appropriately dimensioned matrices $\mathbf{X}=\mathbf{X}^{T} \geq \mathbf{0}$ and $\mathbf{Y}=\mathbf{Y}^{T} \geq \mathbf{0}$, we have the following relations:

$$
\begin{aligned}
0= & d_{2} \boldsymbol{\zeta}_{1}^{T}(k) \mathbf{X} \boldsymbol{\zeta}_{1}(k)-\sum_{l=k-d(k)}^{k-1} \boldsymbol{\zeta}_{1}^{T}(k) \mathbf{X} \boldsymbol{\zeta}_{1}(k) \\
& -\sum_{l=k-d_{2}}^{k-d(k)-1} \zeta_{1}^{T}(k) \mathbf{X} \boldsymbol{\zeta}_{1}(k), \\
0= & \left(d_{2}-d_{1}\right) \zeta_{1}^{T}(k) \mathbf{Y} \boldsymbol{\zeta}_{1}(k)-\sum_{l=k-d(k)}^{k-d_{1}-1} \zeta_{1}^{T}(k) \mathbf{Y} \boldsymbol{\zeta}_{1}(k) \\
& -\sum_{l=k-d_{2}}^{k-d(k)-1} \zeta_{1}^{T}(k) \mathbf{Y} \zeta_{1}(k) .
\end{aligned}
$$

Using (18)-(22), we obtain

$$
\begin{aligned}
\Delta v(\mathbf{x}(k)) \leq \zeta_{2}^{T}(k) \pi \zeta_{2}(k)-\sum_{l=k-d(k)}^{k-1} \zeta_{3}^{T}(k, l) \psi_{1} \zeta_{3}(k, l) \\
-\sum_{l=k-d(k)}^{k-d_{1}-1} \zeta_{3}^{T}(k, l) \psi_{2} \zeta_{3}(k, l) \\
-\sum_{l=k-d_{2}}^{k-d(k)-1} \zeta_{3}^{T}(k, l) \psi_{3} \zeta_{3}(k, l)-\beta
\end{aligned}
$$

where

$$
\begin{aligned}
& \beta=\mathbf{y}^{T}(k) \mathbf{H y}(k)-\mathbf{f}^{T}(\mathbf{y}(k)) \mathbf{H f}(\mathbf{y}(k)), \\
& \pi \\
& =\left[\begin{array}{ccccc}
\boldsymbol{\pi}_{11}+\overline{\mathbf{A}}^{T} \mathbf{H} \overline{\mathbf{A}} & \boldsymbol{\pi}_{12}+\overline{\mathbf{A}}^{T} \mathbf{H} \overline{\mathbf{A}}_{\mathbf{d}} & \mathbf{S}_{1} & -\mathbf{M}_{\mathbf{1}} & -\overline{\mathbf{Z}} \\
\boldsymbol{\pi}_{12}^{T}+\overline{\mathbf{A}}_{\mathbf{d}}^{T} \mathbf{H} \overline{\mathbf{A}} & \boldsymbol{\pi}_{22}+\overline{\mathbf{A}}_{\mathbf{d}}^{T} \mathbf{H} \overline{\mathbf{A}}_{\mathbf{d}} & \mathbf{S}_{2} & -\mathbf{M}_{2} & \mathbf{0} \\
\mathbf{S}_{1}^{T} & \mathbf{S}_{2}^{T} & -\mathbf{Q}_{1} & \mathbf{0} & \mathbf{0} \\
-\mathbf{M}_{1}^{T} & -\mathbf{M}_{2}^{T} & \mathbf{0} & -\mathbf{Q}_{2} & \mathbf{0} \\
-\overline{\mathbf{Z}} & \mathbf{0} & \mathbf{0} & \mathbf{0} & \mathbf{P}-\mathbf{H}+\overline{\mathbf{Z}}
\end{array}\right] \\
& \pi_{11}=-\mathbf{P}+\mathbf{Q}_{1}+\mathbf{Q}_{2}+\left(d_{2}-d_{1}+1\right) \mathbf{Q}_{3}+\overline{\mathbf{Z}}+\mathbf{N}_{\mathbf{1}}+\mathbf{N}_{1}^{\mathrm{T}} \\
& +d_{2} \mathbf{X}_{11}+\left(d_{2}-d_{1}\right) \mathbf{Y}_{11} \\
& \pi_{12}=-\mathbf{N}_{1}+\mathbf{N}_{2}^{\mathrm{T}}+\mathbf{M}_{1}-\mathbf{S}_{1}+d_{2} \mathbf{X}_{12}+\left(d_{2}-d_{1}\right) \mathbf{Y}_{12} \\
& \pi_{22}=-\mathbf{N}_{2}-\mathbf{N}_{2}^{T}+\mathbf{M}_{2}+\mathbf{M}_{2}^{T}-\mathbf{S}_{2}-\mathbf{S}_{2}^{T}-\mathbf{Q}_{3}+d_{2} \mathbf{X}_{22} \\
& +\left(d_{2}-d_{1}\right) \mathbf{Y}_{22} \\
& \overline{\mathbf{A}}=\mathbf{A}+\Delta \mathbf{A}, \quad \overline{\mathbf{A}}_{\mathbf{d}}=\mathbf{A}_{\mathbf{d}}+\Delta \mathbf{A}_{\mathbf{d}},
\end{aligned}
$$

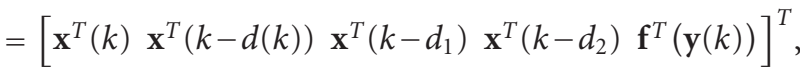

$$
\begin{aligned}
& \zeta_{3}(k, l)=\left[\begin{array}{ll}
\boldsymbol{\zeta}_{1}^{T}(k) & \boldsymbol{\eta}^{T}(l)
\end{array}\right]^{T}
\end{aligned}
$$

In view of Lemma $1,(9)$ implies that the quantity $\beta$ (see $(23 \mathrm{~b}))$ is nonnegative. From (23a), it is clear that $\Delta v(\mathbf{x}(k))<$ 0 if $\boldsymbol{\pi}<\mathbf{0}$ and $(11)-(13)$ hold true and $\Delta v(\mathbf{x}(k))=0$ only when $\zeta_{2}(k)=\mathbf{0}$ and $\boldsymbol{\zeta}_{3}(k, l)=\mathbf{0}$. Thus the conditions $\boldsymbol{\pi}<\mathbf{0}$ and (11) $-(13)$ are sufficient conditions for the global asymptotic stability of system (1a) and (1b)-(4).

By the well-known Schur's complement the condition $\pi<\mathbf{0}$ is equivalent to the following:

$$
\left[\begin{array}{cccccc}
\boldsymbol{\pi}_{11} & \boldsymbol{\pi}_{12} & \mathbf{S}_{\mathbf{1}} & -\mathbf{M}_{\mathbf{1}} & -\overline{\mathbf{Z}} & \overline{\mathbf{A}}^{T} \mathbf{H} \\
\boldsymbol{\pi}_{12}^{T} & \boldsymbol{\pi}_{22} & \mathbf{S}_{2} & -\mathbf{M}_{2} & \mathbf{0} & \overline{\mathbf{A}}_{\mathbf{d}}^{T} \mathbf{H} \\
\mathbf{S}_{1}^{T} & \mathbf{S}_{2}^{T} & -\mathbf{Q}_{1} & \mathbf{0} & \mathbf{0} & \mathbf{0} \\
-\mathbf{M}_{1}^{T} & -\mathbf{M}_{2}^{T} & \mathbf{0} & -\mathbf{Q}_{2} & \mathbf{0} & \mathbf{0} \\
-\overline{\mathbf{Z}} & \mathbf{0} & \mathbf{0} & \mathbf{0} & \mathbf{P}-\mathbf{H}+\overline{\mathbf{Z}} & \mathbf{0} \\
\mathbf{H} \overline{\mathbf{A}} & \mathbf{H} \overline{\mathbf{A}}_{\mathbf{d}} & \mathbf{0} & \mathbf{0} & \mathbf{0} & -\mathbf{H}
\end{array}\right]<\mathbf{0 .}
$$

Now, using (2a), condition (24) can be expressed in the following form:

$$
\mathbf{M}+\overline{\mathbf{B}} \mathbf{F} \overline{\mathbf{C}}+\overline{\mathbf{C}}^{T} \mathbf{F}^{T} \overline{\mathbf{B}}^{T}<\mathbf{0}
$$


where

$$
\begin{gathered}
\mathbf{M}=\left[\begin{array}{cccccc}
\pi_{11} & \pi_{12} & \mathbf{S}_{1} & -\mathbf{M}_{1} & -\overline{\mathbf{Z}} & \mathbf{A}^{T} \mathbf{H} \\
\boldsymbol{\pi}_{12}^{T} & \pi_{22} & \mathbf{S}_{2} & -\mathbf{M}_{2} & \mathbf{0} & \mathbf{A}_{\mathbf{d}}^{T} \mathbf{H} \\
\mathbf{S}_{1}^{T} & \mathbf{S}_{2}^{T} & -\mathbf{Q}_{1} & \mathbf{0} & \mathbf{0} & \mathbf{0} \\
-\mathbf{M}_{1}^{T} & -\mathbf{M}_{2}^{T} & \mathbf{0} & -\mathbf{Q}_{2} & \mathbf{0} & \mathbf{0} \\
-\overline{\mathbf{Z}} & \mathbf{0} & \mathbf{0} & \mathbf{0} & \mathbf{P}-\mathbf{H}+\overline{\mathbf{Z}} & \mathbf{0} \\
\mathbf{H A} & \mathbf{H A}_{\mathbf{d}} & \mathbf{0} & \mathbf{0} & \mathbf{0} & -\mathbf{H}
\end{array}\right] \\
\overline{\mathbf{B}}^{T}=\left[\begin{array}{llllll}
\mathbf{0} & \mathbf{0} & \mathbf{0} & \mathbf{0} & \mathbf{0} & \mathbf{B}^{T} \mathbf{H}
\end{array}\right] \\
\overline{\mathbf{C}}=\left[\begin{array}{lllllll}
\mathbf{C}_{\mathbf{0}} & \mathbf{C}_{1} & \mathbf{0} & \mathbf{0} & \mathbf{0} & \mathbf{0}
\end{array}\right] .
\end{gathered}
$$

By Lemma 2, (25a), (25b), (25c), and (25d) is equivalent to the following:

$$
\mathbf{M}+\varepsilon^{-1} \overline{\mathbf{B}} \overline{\mathbf{B}}^{T}+\varepsilon \overline{\mathbf{C}}^{T} \overline{\mathbf{C}}<\mathbf{0},
$$

where $\varepsilon>0$. The equivalence of (26) and (10) follows trivially from Schur's complement. This completes the proof of Theorem 3.

Remark 4. Theorem 3 provides a stability condition which depends on both the lower delay bound $\left(d_{1}\right)$ and upper delay bound $\left(d_{2}\right)$. For a given $d_{1}\left(d_{2}\right), d_{2}\left(d_{1}\right)$ can be obtained by iteratively solving the inequalities (10)-(13) with respect to $d_{2}\left(d_{1}\right)$.

Remark 5. Pertaining to the case of $L=-1$, the matrix $\mathbf{H}$ in Theorem 3 assumes the form of a positive definite diagonal matrix and consequently, the conditions in Theorem 3 become in true LMI settings. Thus, for $L=-1$, the conditions in Theorem 3 can be easily tested using MATLAB LMI toolbox $[37,38]$.
Remark 6. Note that, in case of $L \neq-1$, the conditions of Theorem 3 are not in true LMI settings. In this case, to determine the global asymptotic stability of system (1a) and (1b)-(4) via Theorem 3, one needs to solve the conditions (10)-(13) for $\varepsilon>0, \mathbf{P}=\mathbf{P}^{T}>\mathbf{0}, \mathbf{Q}_{\mathbf{i}}=\mathbf{Q}_{\mathbf{i}}^{T}>\mathbf{0}(i=1,2,3)$, $\mathbf{Z}_{\mathbf{i}}=\mathbf{Z}_{\mathbf{i}}^{T}>\mathbf{0}(i=1,2), \mathbf{H}=\mathbf{H}^{T}>\mathbf{0}, \mathbf{X} \geq \mathbf{0}, \mathbf{Y} \geq \mathbf{0}$, $\mathbf{N}, \mathbf{M}$ and $\mathbf{S}$ using MATLAB LMI toolbox $[37,38]$ and also check if there exists a solution $\mathbf{H}=\mathbf{H}^{T}>\mathbf{0}$ meeting (9). This method essentially involves repeated searching of $\varepsilon>0$, $\mathbf{P}=\mathbf{P}^{T}>\mathbf{0}, \mathbf{Q}_{\mathbf{i}}=\mathbf{Q}_{\mathbf{i}}^{T}>\mathbf{0}(i=1,2,3), \mathbf{Z}_{\mathbf{i}}=\mathbf{Z}_{\mathbf{i}}^{T}>\mathbf{0}(i=1,2)$, $\mathbf{H}=\mathbf{H}^{T}>\mathbf{0}, \mathbf{X} \geq \mathbf{0}, \mathbf{Y} \geq \mathbf{0}, \mathbf{N}, \mathbf{M}$ and $\mathbf{S}$ satisfying (10)-(13) until a solution $\mathbf{H}=\mathbf{H}^{T}>\mathbf{0}$ meeting (9) is found. If (9)-(13) provide a feasible solution, then the system (1a) and (1b)(4) is globally asymptotically stable; otherwise, no conclusion regarding the global asymptotic stability of the system under consideration can be drawn.

In the following, as an extension of the present approach, we develop a criterion which is true LMI-based and computationally simpler than Theorem 3.

Let the matrix $\mathbf{H}=\left[h_{i j}\right] \in \mathbf{R}^{n \times n}$ is represented by

$$
\begin{gathered}
h_{i i}=g_{i}+\sum_{j=1, j \neq i}^{n}\left(\alpha_{i j}+\beta_{i j}\right), \quad i=1,2, \ldots, n, \\
h_{i j}=h_{j i}=\left(\frac{1+L}{2}\right)\left(\alpha_{i j}-\beta_{i j}\right), \quad i, j=1,2, \ldots, n(27 \mathrm{a}) \\
\left.\alpha_{i j}=\alpha_{j i}>0, \quad i \neq j\right), \\
\beta_{i j}=\beta_{j i}>0, \quad i, j=1,2, \ldots, n \underset{(27 \mathrm{~b})}{(i \neq j),}(27 \mathrm{c}) \\
g_{i}>0, \quad i=1,2, \ldots, n,
\end{gathered}
$$

where it is understood that, for $n=1, \mathbf{H}$ corresponds to a scalar $\gamma>0$. Thus, corresponding to $n=3$, the matrix $\mathbf{H}$ takes the form

$$
\mathbf{H}=\left[\begin{array}{ccc}
g_{1}+\alpha_{12}+\beta_{12}+\alpha_{13}+\beta_{13} & \left(\frac{1+L}{2}\right)\left(\alpha_{12}-\beta_{12}\right) & \left(\frac{1+L}{2}\right)\left(\alpha_{13}-\beta_{13}\right) \\
\left(\frac{1+L}{2}\right)\left(\alpha_{12}-\beta_{12}\right) & g_{2}+\alpha_{12}+\beta_{12}+\alpha_{23}+\beta_{23} & \left(\frac{1+L}{2}\right)\left(\alpha_{23}-\beta_{23}\right) \\
\left(\frac{1+L}{2}\right)\left(\alpha_{13}-\beta_{13}\right) & \left(\frac{1+L}{2}\right)\left(\alpha_{23}-\beta_{23}\right) & g_{3}+\alpha_{13}+\beta_{13}+\alpha_{23}+\beta_{23}
\end{array}\right],
$$

where $g_{i}>0, \alpha_{i j}=\alpha_{j i}>0, \beta_{i j}=\beta_{j i}>0, i, j=$ $1,2,3(i \neq j)$ and $L \in[-1,1]$.

It can be verified that the matrix $\mathbf{H}$ represented by $(27 \mathbf{a})$, (27b), (27c), and (27d) is a positive definite symmetric matrix and satisfies (9). Further, any positive definite symmetric matrix $\mathbf{H}$ satisfying (9) can always be expressed in the form of (27a), (27b), (27c), and (27d). Consequently, Theorem 3 can equivalently be stated as follows.

Theorem 7. For given positive integers $d_{1}$ and $d_{2}$ with $d_{2} \geq$ $d_{1}$, the system described by (1a) and (1b)-(4) is globally asymptotically stable if there exists appropriately dimensioned matrices $\mathbf{P}=\mathbf{P}^{T}>\mathbf{0}, \mathbf{Q}_{\mathbf{i}}=\mathbf{Q}_{\mathbf{i}}{ }^{T}>\mathbf{0}(i=1,2,3)$, $\mathbf{Z}_{\mathbf{i}}=\mathbf{Z}_{\mathbf{i}}{ }^{T}>\mathbf{0}(i=1,2), \mathbf{X}=\left[\begin{array}{ll}\mathbf{X}_{11} & \mathbf{X}_{12} \\ \mathbf{X}_{12}{ }^{T} & \mathbf{X}_{22}\end{array}\right] \geq \mathbf{0}, \mathbf{Y}=$ $\left[\begin{array}{ll}\mathbf{Y}_{11} & \mathbf{Y}_{12} \\ \mathbf{Y}_{12}{ }^{T} & \mathbf{Y}_{22}\end{array}\right] \geq \mathbf{0}, \mathbf{N}=\left[\begin{array}{l}\mathbf{N}_{1} \\ \mathbf{N}_{2}\end{array}\right], \mathbf{M}=\left[\begin{array}{l}\mathbf{M}_{1} \\ \mathbf{M}_{2}\end{array}\right], \mathbf{S}=\left[\begin{array}{l}\mathbf{S}_{1} \\ \mathbf{S}_{2}\end{array}\right]$ and scalars $\mathcal{E}>0, g_{i}>0(i=1,2, \ldots, n), \alpha_{i j}=\alpha_{j i}>0$, $\beta_{i j}=\beta_{j i}>0(i, j=1,2, \ldots, n(i \neq j))$ satisfying $(10)-(13)$ where $\mathbf{H}$ is characterized by (27a), (27b), (27c), and (27d).

Remark 8. For $L=-1$, the matrix $\mathbf{H}$ in Theorem 7 (or Theorem 3 ) reduces to a positive definite diagonal matrix and consequently, the conditions in Theorem 7 (or 
Theorem 3) become in true LMI settings. However, for $L \neq-1$, Theorem 7 provides true LMI conditions for global asymptotic stability, without a need for searching $\mathbf{H}$ meeting (9) as in Theorem 3 (see Remark 6), which is beneficial in terms of numerical complexity. It may be observed that, for $L \neq-1$, the matrix inequalities in Theorem 7 become linear in the variables $\mathbf{P}, \mathbf{Q}_{\mathbf{i}}(i=1,2,3), \mathbf{Z}_{\mathbf{i}}$ $(i=1,2), \mathbf{X}, \mathbf{Y}, \mathbf{N}, \mathbf{M}, \mathbf{S}, \varepsilon, g_{i}(i=1,2, \ldots, n), \alpha_{i j}, \beta_{i j}(i, j=$ $1,2, \ldots, n(i \neq j))$ and, thus, are computationally tractable. Since the matrix $\mathbf{H}$ described by (27a), (27b), (27c), and (27d) has a built-in feature of satisfying (9), one needs not bother about (9) while using Theorem 7.

In the case of constant delay, the lower and the upper delay bounds in (4) becomes identical (i.e., $d_{1}=d_{2}=d$ ) and Theorem 7 leads to the following corollary.

Corollary 9. The system (1a) and (1b)-(4) with $d(k)=d$ is globally asymptotically stable if there exists appropriately dimensioned matrices $\mathbf{P}=\mathbf{P}^{T}>\mathbf{0}, \mathbf{Q}_{\mathbf{i}}=\mathbf{Q}_{\mathbf{i}}^{T}>\mathbf{0}(i=$ $1,2,3), \mathbf{Z}_{\mathbf{i}}=\mathbf{Z}_{\mathbf{i}}^{T}>\mathbf{0}(i=1,2), \mathbf{X}=\left[\begin{array}{ll}\mathbf{X}_{11} & \mathbf{X}_{12} \\ \mathbf{X}_{12}^{T} & \mathbf{X}_{22}\end{array}\right] \geq \mathbf{0}$, $\mathbf{Y}=\left[\begin{array}{ll}\mathbf{Y}_{11} & \mathbf{Y}_{12} \\ \mathbf{Y}_{12}^{T} \mathbf{Y}_{22}\end{array}\right] \geq \mathbf{0}, \mathbf{N}=\left[\begin{array}{l}\mathbf{N}_{1} \\ \mathbf{N}_{2}\end{array}\right], \mathbf{M}=\left[\begin{array}{l}\mathbf{M}_{1} \\ \mathbf{M}_{2}\end{array}\right], \mathbf{S}=\left[\begin{array}{l}\mathrm{S}_{1} \\ \mathrm{~S}_{2}\end{array}\right]$, scalars $\varepsilon>0, g_{i}>0(i=1,2, \ldots, n), \alpha_{i j}=\alpha_{j i}>0$, $\beta_{i j}=\beta_{j i}>0(i, j=1,2, \ldots, n(i \neq j))$ satisfying (11)-(13) and$$
\left[\begin{array}{ccccccc}
\hat{\xi}_{11} & \hat{\xi}_{12} & \mathbf{S}_{1} & -\mathbf{M}_{1} & -d \mathbf{Z}_{1} & \mathbf{A}^{T} \mathbf{H} & \mathbf{0} \\
\hat{\xi}_{12}^{T} & \hat{\xi}_{22} & \mathbf{S}_{2} & -\mathbf{M}_{2} & \mathbf{0} & \mathbf{A}_{\mathbf{d}}^{T} \mathbf{H} & \mathbf{0} \\
\mathbf{S}_{1}^{T} & \mathbf{S}_{2}^{T} & -\mathbf{Q}_{1} & \mathbf{0} & \mathbf{0} & \mathbf{0} & \mathbf{0} \\
-\mathbf{M}_{1}^{T} & -\mathbf{M}_{2}^{T} & \mathbf{0} & -\mathbf{Q}_{2} & \mathbf{0} & \mathbf{0} & \mathbf{0} \\
-d \mathbf{Z}_{1} & \mathbf{0} & \mathbf{0} & \mathbf{0} & \mathbf{P}-\mathbf{H}+d \mathbf{Z}_{1} & \mathbf{0} & \mathbf{0} \\
\mathbf{H A} & \mathbf{H} \mathbf{A}_{\mathbf{d}} & \mathbf{0} & \mathbf{0} & \mathbf{0} & -\mathbf{H} & \mathbf{H B} \\
\mathbf{0} & \mathbf{0} & \mathbf{0} & \mathbf{0} & \mathbf{0} & \mathbf{B}^{T} \mathbf{H} & -\varepsilon \mathbf{I}
\end{array}\right]<\mathbf{0}
$$

where

$$
\begin{aligned}
\hat{\xi}_{11}= & -\mathbf{P}+\mathbf{Q}_{1}+\mathbf{Q}_{2}+\mathbf{Q}_{3}+d \mathbf{Z}_{1}+\mathbf{N}_{1}+\mathbf{N}_{1}^{T} \\
& +d \mathbf{X}_{11}+\varepsilon \mathbf{C}_{0}^{T} \mathbf{C}_{0}, \\
\hat{\xi}_{12}= & -\mathbf{N}_{1}+\mathbf{N}_{2}^{T}+\mathbf{M}_{1}-\mathbf{S}_{1}+d \mathbf{X}_{12}+\varepsilon \mathbf{C}_{0}^{T} \mathbf{C}_{1}, \\
\widehat{\xi}_{22}= & -\mathbf{N}_{2}-\mathbf{N}_{2}^{T}+\mathbf{M}_{2}+\mathbf{M}_{2}^{T}-\mathbf{S}_{2}-\mathbf{S}_{2}^{T}-\mathbf{Q}_{3} \\
& +d \mathbf{X}_{22}+\varepsilon \mathbf{C}_{1}^{T} \mathbf{C}_{1},
\end{aligned}
$$

and $\mathbf{H}$ is given by (27a), (27b), (27c), and (27d).

\section{Comparison with A Previous Work [18]}

A delay-independent criterion for the global asymptotic stability of a class of uncertain discrete-time systems involving multiple state delays and generalized overflow nonlinearities is reported in [18]. In this section, for the case of single delay, it will be shown how the delay-independent stability criterion [18, Theorem 1] is recovered from Corollary 9 as a special case.

Following the proof of [18, Theorem 1], one can easily see that the system (1a) and (1b)-(4) with $d(k)=d$ is globally asymptotically stable if there exists a positive scalar $\varepsilon$ and $n \times n$ positive definite symmetric matrices $\mathbf{P}, \mathbf{Q}$, and $\mathbf{H}$ such that

$$
\left[\begin{array}{ccccc}
-\mathbf{P}+\mathbf{Q}+\varepsilon \mathbf{C}_{0}^{T} \mathbf{C}_{0} & \varepsilon \mathbf{C}_{0}^{T} \mathbf{C}_{1} & \mathbf{0} & \mathbf{A}^{T} \mathbf{H} & \mathbf{0} \\
\varepsilon \mathbf{C}_{1}^{T} \mathbf{C}_{0} & -\mathbf{Q}+\varepsilon \mathbf{C}_{1}^{T} \mathbf{C}_{1} & \mathbf{0} & \mathbf{A}_{\mathbf{d}}^{T} \mathbf{H} & \mathbf{0} \\
\mathbf{0} & \mathbf{0} & \mathbf{P}-\mathbf{H} & \mathbf{0} & \mathbf{0} \\
\mathbf{H A} & \mathbf{H A}_{\mathbf{d}} & \mathbf{0} & -\mathbf{H} & \mathbf{H B} \\
\mathbf{0} & \mathbf{0} & \mathbf{0} & \mathbf{B}^{T} \mathbf{H} & -\varepsilon \mathbf{I}
\end{array}\right]<\mathbf{0},
$$

where $\mathbf{H}$ is defined by (27a), (27b), (27c), and (27d).

By choosing the parameters $\mathbf{X}_{12}=\mathrm{Y}_{12}=\mathbf{N}=\mathbf{M}=$ $\mathbf{S}=\mathbf{0}, \mathbf{Q}_{1}=\rho_{1} \mathbf{I}, \mathbf{Q}_{2}=\rho_{2} \mathbf{I}, \mathbf{Q}_{3}=\mathbf{Q}, \mathbf{Z}_{1}=\rho_{3} \mathbf{I} / d, \mathbf{Z}_{2}=$ $\rho_{4} \mathbf{I} / d, \mathbf{X}_{11}=\rho_{5} \mathbf{I} / d, \mathbf{X}_{22}=\rho_{6} \mathbf{I} / d, \mathbf{Y}_{11}=\rho_{7} \mathbf{I} / d, \mathbf{Y}_{22}=\rho_{8} \mathbf{I} / d$, for some sufficiently small positive scalars $\rho_{i}(i=1,2, \ldots, 8)$, the conditions in Corollary 9 reduces to (31). Thus, for the case of single delay, the delay-independent stability criterion $[18$, Theorem 1] is recovered from Corollary 9 as a special case.

\section{An Illustrative Example}

Consider a system represented by (1a) and (1b)-(4) with

$$
\begin{array}{cc}
L=-1, \quad \mathbf{A}=\left[\begin{array}{cc}
0.8 & 0 \\
0.05 & 0.9
\end{array}\right], \quad \mathbf{A}_{\mathbf{d}}=\left[\begin{array}{cc}
0.01 & 0 \\
0 & 0.02
\end{array}\right], \\
\mathbf{B}=\left[\begin{array}{ll}
0 & 0.1
\end{array}\right]^{T}, \quad \mathbf{C}_{0}=\left[\begin{array}{ll}
0.01 & 0
\end{array}\right], \quad \mathbf{C}_{\mathbf{1}}=\left[\begin{array}{ll}
0 & 0.01
\end{array}\right] .
\end{array}
$$

Let us select $d_{1}=2$ and $d_{2}=10$. With the help of Matlab LMI toolbox [37, 38], it is found that (10)-(13) are feasible for the following values of unknown parameters.

$$
\begin{aligned}
\mathbf{P} & =\left[\begin{array}{ll}
834.51 & 5.7678 \\
5.7678 & 569.3174
\end{array}\right], \\
\mathbf{Q}_{1} & =\left[\begin{array}{cc}
23.1690 & -2.2272 \\
-2.2272 & 4.3780
\end{array}\right], \\
\mathbf{Q}_{2} & =\left[\begin{array}{cc}
23.4337 & -2.2530 \\
-2.2530 & 4.4249
\end{array}\right], \\
\mathbf{Q}_{3} & =\left[\begin{array}{cc}
9.8871 & -0.7648 \\
-0.7648 & 4.4261
\end{array}\right],
\end{aligned}
$$




$$
\begin{aligned}
& \mathrm{Z}_{1}=\left[\begin{array}{cc}
1.1158 & -0.1113 \\
-0.1113 & 0.1791
\end{array}\right], \\
& \mathrm{Z}_{2}=\left[\begin{array}{cc}
1.5860 & -0.1572 \\
-0.1572 & 0.2643
\end{array}\right] \text {, } \\
& \mathbf{X}=\left[\begin{array}{cccc}
3.5791 & -0.2228 & -0.3348 & -0.0213 \\
-0.2228 & 1.5497 & 0.0043 & -0.4386 \\
-0.3348 & 0.0043 & 0.5415 & -0.0451 \\
-0.0213 & -0.4386 & -0.0451 & 0.1951
\end{array}\right] \\
& \mathbf{Y}=\left[\begin{array}{cccc}
4.6378 & -0.2861 & -0.3943 & -0.0326 \\
-0.2861 & 2.0289 & 0.0010 & -0.5704 \\
-0.3943 & 0.0010 & 0.7649 & -0.0650 \\
-0.0326 & -0.5704 & -0.0650 & 0.2606
\end{array}\right] \text {, } \\
& \mathbf{N}=\left[\begin{array}{cc}
-0.2430 & 0.0242 \\
0.0241 & -0.0401 \\
0.2682 & -0.0269 \\
-0.0269 & 0.0414
\end{array}\right] \\
& \mathbf{M}=\left[\begin{array}{cc}
0.0149 & -0.0008 \\
-0.0010 & 0.0059 \\
-1.1023 & 0.1103 \\
0.1102 & -0.1711
\end{array}\right] \text {, } \\
& \mathbf{S}=\left[\begin{array}{cc}
0.0047 & -0.0004 \\
-0.0005 & 0.0006 \\
0.4861 & -0.0487 \\
-0.0485 & 0.0764
\end{array}\right] \text {, } \\
& \mathbf{H}=\left[\begin{array}{cc}
891.2363 & 0 \\
0 & 577.5832
\end{array}\right] \text {, } \\
& \varepsilon=356.1845 .
\end{aligned}
$$

Therefore, according to Theorem 3 , the system under consideration is globally asymptotically stable. Further, by selecting $d_{1}=2$ and iteratively solving (10)-(13) with respect to $d_{2}$, it is seen that the system (1a) and (1b)-(4), (32) is also globally asymptotically stable for $2 \leq d(k) \leq 23$.

\section{Conclusions}

A new computationally tractable delay-dependent stability criterion for a class of uncertain discrete-time systems with time-varying delay subject to generalized overflow nonlinearities has been established. As shown in Section 4, pertaining to the systems involving single delay, the delayindependent stability criterion [18, Theorem 1] has been recovered from Corollary 9 as a special case. The effectiveness of the results presented has been illustrated with a numerical example. The results discussed in this paper can easily be extended to a class of nonlinear uncertain discrete-time systems involving multiple state delays.

\section{Abbreviations}

$\mathbf{R}^{p \times q}$ : Set of $p \times q$ real matrices

$\mathbf{R}^{p}$ : $\quad$ Set of $p \times 1$ real vectors

0: $\quad$ Null matrix or null vector of appropriate dimension

I: $\quad$ Identity matrix of appropriate dimension

$\mathbf{G}^{\mathrm{T}}$ : $\quad$ Transpose of the matrix (or vector) $\mathbf{G}$

$\mathbf{G}>\mathbf{0}$ : $\mathbf{G}$ is positive definite symmetric matrix

$\mathbf{G} \geq \mathbf{0}$ : $\mathbf{G}$ is positive semidefinite symmetric matrix

$\mathbf{G}<\mathbf{0}$ : $\mathbf{G}$ is negative definite symmetric matrix.

\section{References}

[1] T. Bose and M. Q. Chen, "Overflow oscillations in state-space digital filters," IEEE Transactions on Circuits and Systems, vol. 38, no. 7, pp. 807-810, 1991.

[2] J. H. F. Ritzerfeld, "A condition for the overflow stability of second-order digital filters that is satisfied by all scaled state-space structures using saturation," IEEE Transactions on Circuits and Systems, vol. 36, no. 8, pp. 1049-1057, 1989.

[3] V. Singh, "Elimination of overflow oscillations in fixed-point state-space digital filters using saturation arithmetic," IEEE Transactions on Circuits and Systems, vol. 37, no. 6, pp. 814818, 1990.

[4] V. Singh, "A new frequency-domain criterion for elimination of limit cycles in fixed-point state-space digital filters using saturation arithmetic," Chaos, Solitons \& Fractals, vol. 34, no. 3, pp. 813-816, 2007.

[5] H. Kar and V. Singh, "A new criterion for the overflow stability of second-order state-space digital filters using saturation arithmetic," IEEE Transactions on Circuits and Systems I, vol. 45, no. 3, pp. 311-313, 1998.

[6] V. Singh, "A new realizability condition for limit cycle free state-space digital filter employing saturation arithmetic," IEEE Transactions on Circuits and Systems, vol. 32, no. 10, pp. 1070-1071, 1985.

[7] H. Kar and V. Singh, "Elimination of overflow oscillations in fixed-point state-space digital filters with saturation arithmetic: an LMI approach," IEEE Transactions on Circuits and Systems II, vol. 51, no. 1, pp. 40-42, 2004.

[8] D. Liu and A. N. Michel, "Asymptotic stability of discretetime systems with saturation nonlinearities with applications to digital filters," IEEE Transactions on Circuits and Systems I, vol. 39, no. 10, pp. 798-807, 1992.

[9] H. Kar and V. Singh, "Robust stability of 2-D discrete systems described by the Fornasini-Marchesini second model employing quantization/overflow nonlinearities," IEEE Transactions on Circuits and Systems II, vol. 51, no. 11, pp. 598-602, 2004.

[10] H. Kar and V. Singh, "Elimination of overflow oscillations in digital filters employing saturation arithmetic," Digital Signal Processing, vol. 15, no. 6, pp. 536-544, 2005.

[11] V. Singh, "Modified form of Liu-Michel's criterion for global asymptotic stability of fixed-point state-space digital filters using saturation arithmetic," IEEE Transactions on Circuits and Systems II, vol. 53, no. 12, pp. 1423-1425, 2006.

[12] H. Kar, "An LMI based criterion for the nonexistence of overflow oscillations in fixed-point state-space digital filters using saturation arithmetic," Digital Signal Processing, vol. 17, no. 3, pp. 685-689, 2007.

[13] H. Kar, "An improved version of modified Liu-Michel's criterion for global asymptotic stability of fixed-point statespace digital filters using saturation arithmetic," Digital Signal Processing, vol. 20, no. 4, pp. 977-981, 2010. 
[14] V. Singh, "Modified criterion for global asymptotic stability of fixed-point state-space digital filters using two's complement arithmetic," Automatica, vol. 46, no. 2, pp. 475-478, 2010.

[15] H. Kar, "Comments on "modified criterion for global asymptotic stability of fixed-point state-space digital filters using two's complement arithmetic" [Automatica 46 (2010) 475478]," Automatica, vol. 46, no. 11, pp. 1925-1927, 2010.

[16] H. Kar, "Asymptotic stability of fixed-point state-space digital filters with combinations of quantization and overflow nonlinearities," Signal Processing, vol. 91, no. 11, pp. 2667-2670, 2011.

[17] S. F. Chen, "Asymptotic stability of discrete-time systems with time-varying delay subject to saturation nonlinearities," Chaos, Solitons \& Fractals, vol. 42, no. 2, pp. 1251-1257, 2009.

[18] V. Krishna Rao Kandanvli and H. Kar, "Robust stability of discrete-time state-delayed systems employing generalized overflow nonlinearities," Nonlinear Analysis: Theory, Methods ¿ Applications, vol. 69, no. 9, pp. 2780-2787, 2008.

[19] V. Krishna Rao Kandanvli and H. Kar, "Robust stability of discrete-time state-delayed systems with saturation nonlinearities: linear matrix inequality approach," Signal Processing, vol. 89, no. 2, pp. 161-173, 2009.

[20] V. Krishna Rao Kandanvli and H. Kar, "An LMI condition for robust stability of discrete-time state-delayed systems using quantization/overflow nonlinearities," Signal Processing, vol. 89, no. 11, pp. 2092-2102, 2009.

[21] V. K. R. Kandanvli and H. Kar, "Delay-dependent LMI condition for global asymptotic stability of discrete-time uncertain state-delayed systems using quantization/overflow nonlinearities," International Journal of Robust and Nonlinear Control, vol. 21, no. 14, pp. 1611-1622, 2011.

[22] T. Ooba, "Stability of linear discrete dynamics employing state saturation arithmetic," IEEE Transactions on Automatic Control, vol. 48, no. 4, pp. 626-630, 2003.

[23] Y. He, M. Wu, G. P. Liu, and J. H. She, "Output feedback stabilization for a discrete-time system with a time-varying delay," IEEE Transactions on Automatic Control, vol. 53, no. 10, pp. 2372-2377, 2008.

[24] Y. He, G. P. Liu, D. Rees, and M. Wu, "Hळ filtering for discretetime systems with time-varying delay," Signal Processing, vol. 89, no. 3, pp. 275-282, 2009.

[25] S. Xu and J. Lam, "On equivalence and efficiency of certain stability criteria for time-delay systems," IEEE Transactions on Automatic Control, vol. 52, no. 1, pp. 95-101, 2007.

[26] S. Xu and J. Lam, "A survey of linear matrix inequality techniques in stability analysis of delay systems," International Journal of Systems Science, vol. 39, no. 12, pp. 1095-1113, 2008.

[27] H. Gao, J. Lam, C. Wang, and Y. Wang, "Delay-dependent output-feedback stabilisation of discrete-time systems with time-varying state delay," IEE Proceedings on Control Theory and Applications, vol. 151, no. 6, pp. 691-698, 2004.

[28] H. Gao and T. Chen, "New results on stability of discrete-time systems with time-varying state delay," IEEE Transactions on Automatic Control, vol. 52, no. 2, pp. 328-334, 2007.

[29] X. Ji, T. Liu, Y. Sun, and H. Su, "Stability analysis and controller synthesis for discrete linear time-delay systems with state saturation nonlinearities," International Journal of Systems Science, vol. 42, no. 3, pp. 397-406, 2011.

[30] Y. He, Q. G. Wang, C. Lin, and M. Wu, "Delay-rangedependent stability for systems with time-varying delay," Automatica, vol. 43, no. 2, pp. 371-376, 2007.

[31] J. Qiu, G. Feng, and J. Yang, "Improved delay-dependent $\mathrm{H} \infty$ filtering design for discrete-time polytopic linear delay systems," IEEE Transactions on Circuits and Systems II, vol. 55, no. 2, pp. 178-182, 2008.

[32] L. Xie, M. Fu, and C. E. de Souza, " $\mathrm{H} \infty$ control and quadratic stabilization of systems with parameter uncertainty via output feedback," IEEE Transactions on Automatic Control, vol. 37, no. 8, pp. 1253-1256, 1992.

[33] F. Yang and Y. S. Hung, "Robust mixed $\mathrm{H} 2 / \mathrm{H} \infty$ filtering with regional pole assignment for uncertain discrete-time systems," IEEE Transactions on Circuits and Systems I, vol. 49, no. 8, pp. 1236-1241, 2002.

[34] S. Xu, J. Lam, Z. Lin, and K. Galkowski, "Positive real control for uncertain two-dimensional systems," IEEE Transactions on Circuits and Systems I, vol. 49, no. 11, pp. 1659-1666, 2002.

[35] F. Yang, Z. Wang, Y. S. Hung, and M. Gani, "Hळ control for networked systems with random communication delays," IEEE Transactions on Automatic Control, vol. 51, no. 3, pp. 511-518, 2006.

[36] X. He, Z. Wang, and D. Zhou, "Robust $\mathrm{H} \infty$ filtering for networked systems with multiple state delays," International Journal of Control, vol. 80, no. 8, pp. 1217-1232, 2007.

[37] S. Boyd, L. El Ghaoui, E. Feron, and V. Balakrishnan, Linear Matrix Inequalities in System and Control Theory, SIAM, Philadelphia, PA, USA, 1994.

[38] P. Gahinet, A. Nemirovski, A. J. Laub, and M. Chilali, LMI Control Toolbox-for Use With Matlab, The MATH Works, Natic, Mass, USA, 1995. 


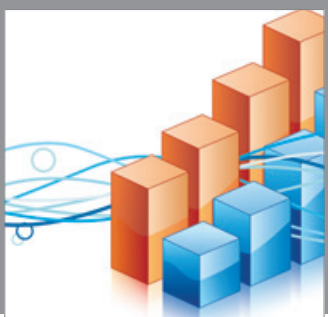

Advances in

Operations Research

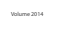

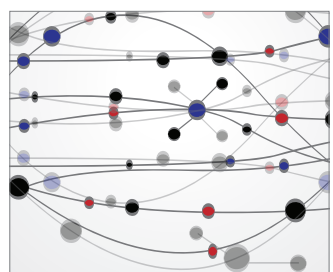

\section{The Scientific} World Journal
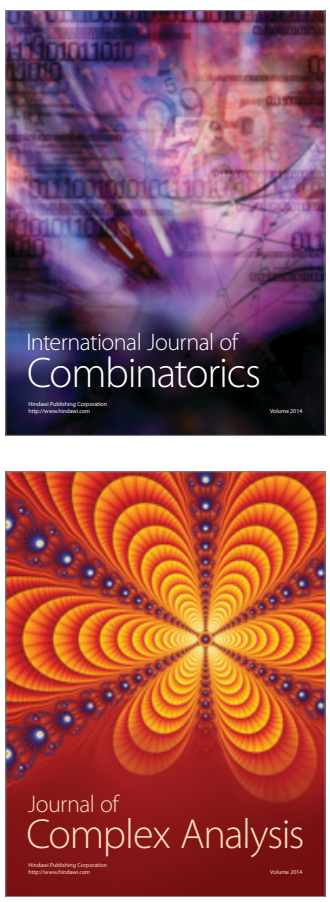

International Journal of

Mathematics and

Mathematical

Sciences
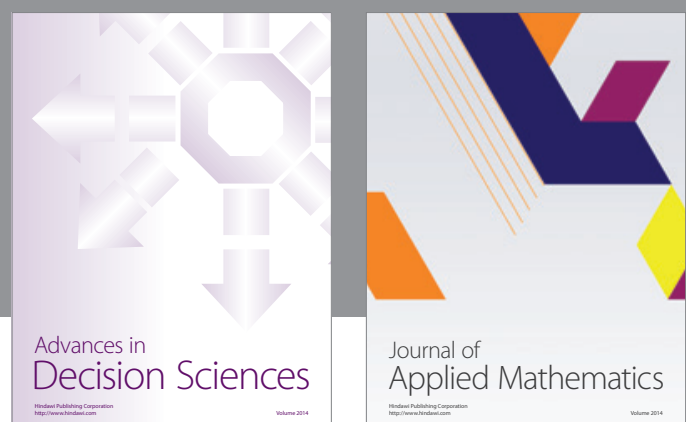

Journal of

Applied Mathematics
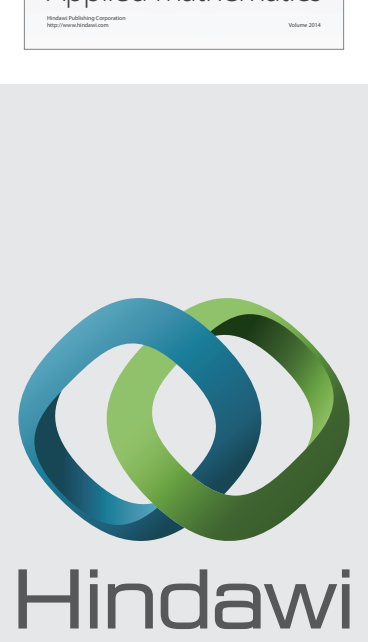

Submit your manuscripts at http://www.hindawi.com
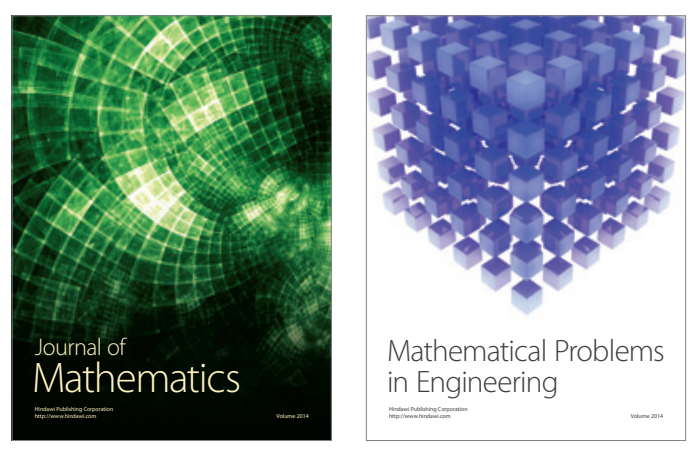

Mathematical Problems in Engineering
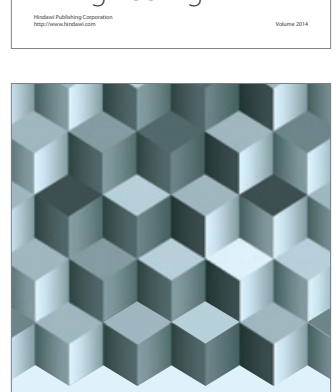

Journal of

Function Spaces
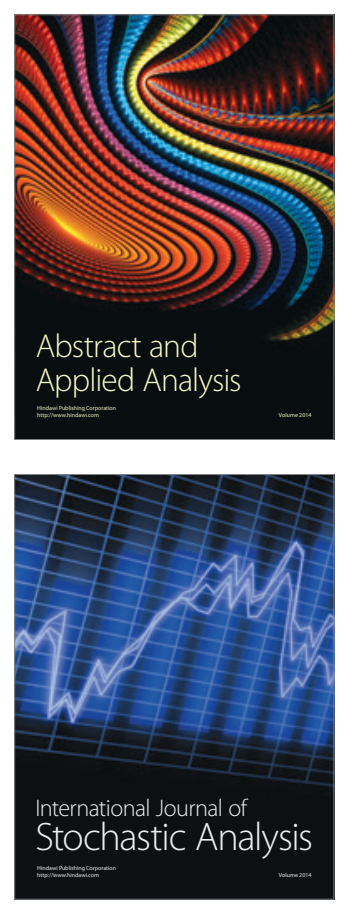

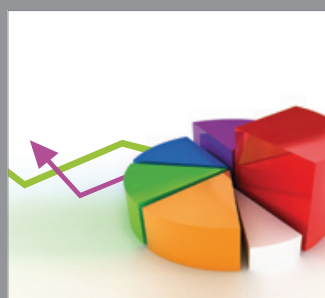

ournal of

Probability and Statistics

Promensencen
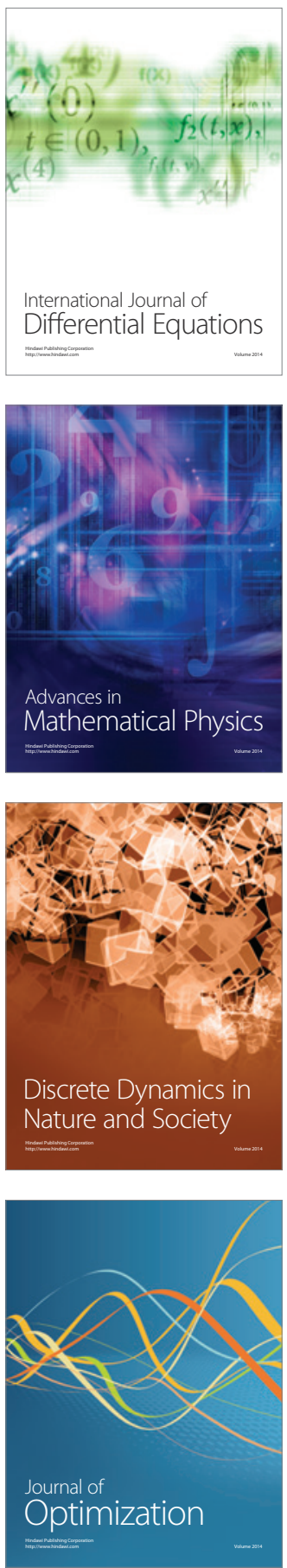